\title{
La integración del análisis cualitativo y cuantitativo en la evaluación de un programa familiar en procesos de emancipación juvenil
}

\author{
Lluís Ballester ${ }^{1}$, Carlos Rosón ${ }^{2}$, Victòria Quesada ${ }^{1}$, Albert \\ Cabellos ${ }^{1}$ \\ ${ }^{1}$ Departamento de Pedagogía y Didácticas Específicas, Universitat de les \\ Illes Balears, España | lluis.ballester@uib.es | https://orcid.org/ 0000-0003 \\ 1861-7511 | victoria.quesada@uib.eu | https://orcid.org/0000-0002-8881- \\ 2358 | albert.cabellos@uib.cat | https://orcid.org/ 0000-0001-5738-8358 \\ ${ }^{2}$ Escuela de Trabajo Social, Universidad de Santiago de Compostela; Centro FAIA de \\ Documentación e Investigación Social, España | carlos.roson@igaxes.org | https://orcid.org/ \\ 0000-0001-6513-1720
}

\begin{abstract}
Resumen: La combinación de enfoques de análisis cuantitativos y cualitativos es una realidad de la investigación avanzada en las ciencias sociales. El objetivo de este trabajo es evaluar la eficacia, mediante integración de métodos, de un programa para mejorar la parentalidad, la dinámica familiar, así como las conductas de riesgo de los adolescentes. Se aplica un diseño cuasi-experimental con grupos de control, basado en toma de datos cuantitativos (centrados en resultados) y cualitativos (aquí centrados en el proceso), en el mismo diseño. La investigación cuantitativa, basada en modelos experimentales, ofrece medidas contrastables y replicables que permitan contrastar hipótesis. Los enfoques cualitativos se orientan a la comprensión o al cambio de realidades, ofreciendo análisis de informaciones elaboradas por los sujetos a partir de metodologías diversas (entrevista, grupos focales, etc.). Se ha conseguido identificar los cambios significativos, así como la información cualitativa contextual que mejora su comprensión: reducción del conflicto familiar, mejora de la parentalidad positiva, mejora de la resiliencia, mejora de la implicación familiar.
\end{abstract}

Palabras Clave: Emanicipación juvenil; Programas de intervención; Evaluación de Efectividad; Métodos mixtos

\section{Introducción - El Reto de la Integración de Métodos}

La integración de métodos permite combinar lo mejor de dos mundos, articulando hipótesis y preguntas de investigación, metodologías basadas en instrumentos validados y metodologías basadas en la escucha o la captación del dato complejo (Brannen, 2017; Halcomb \& Hickman, 2015). A continuación, se presentan los tópicos más destacados para identificar las características diferenciales de la integración de métodos.

Una de las mejoras, para el análisis, que ofrece el enfoque integrado es la posibilidad de combinar datos e informaciones, escalas nominales, ordinales, de intervalo y de razón, junto a informaciones que difícilmente se someten a la disciplina de las escalas de medida. Los datos son tomados a partir de instrumentos de medida y las informaciones a partir de registros complejos. Con este enfoque se dispone de variables medidas con precisión y estructuradas en hipótesis; pero, también de categorías de análisis estructuradas en preguntas de investigación (Jason \& Glenwick, 2016).

Se pueden combinar muestras estadísticas con muestras por cuotas. Las primeras para poder dimensionar los fenómenos objeto de estudio; las segundas para poder profundizar y seguir a lo largo de tiempos más dilatados. El tratamiento de muestras mixtas no se ha desarrollado de manera continuada, pero se dispone de suficientes ejemplos como para saber bien cómo procesarlas de manera combinada (Ballester, Nadal, \& Amer, 2017; Creswell \& Creswell, 2017; León \& Montero, 2020).

Si la metodología experimental se caracteriza por el control, las investigaciones mediante cuestionarios u observación sistemática también disponen de rigurosos protocolos. La investigación cualitativa permite controles por triangulación, saturación y otros (Denzin \& Lincoln, 2011). Con el uso de herramientas informáticas, como NVivo (QSR) y las actuales 
técnicas de recogida y obtención de información, la investigación puede trabajar con fuentes de información diversas. Se puede integrar el análisis de documentación (informes y documentación gris, artículos, publicaciones) directamente con los registros audiovisuales o con transcripciones de entrevistas semiestructuradas, grupos de discusión, observación participante u otra información. Pero, también se pueden combinar con los datos que provienen de cuestionarios o registros cuasi-experimentales, por lo que permiten combinar diversas fuentes de datos para estudiar a fondo las preguntas de investigación realizadas (Brannen, 2017).

Habitualmente, en investigación cualitativa, por ejemplo, etnográfica, documental o narrativa, se considera que en el análisis cualitativo no se trabaja con estadísticas (Gibbs, 2012; Miles, Huberman, \& Saldaña, 2014; Rapley, 2014), sin embargo, se puede trabajar con una gama amplia de estadísticas no paramétricas que permiten completar análisis inicialmente más narrativos o interpretativos. Por ejemplo, se pueden introducir análisis de concordancia entre informantes (Kappa de Cohen, por ejemplo), análisis de asociación entre categorías (Chi cuadrado) o análisis multivariables complejos basados en frecuencias de opciones (análisis de correspondencias). Dichos análisis permiten completar, desde enfoques cuantitativos, las interpretaciones cualitativas (Wyse, Selwyn, Smith, \& Suter, 2016). Reservaremos los análisis paramétricos para las muestras estadísticas y los enfoques inferenciales.

Estos y otros desarrollos han permitido un aumento considerable de las investigaciones basadas en integración de métodos. Desde una perspectiva aplicada y centrada en los procesos de emancipación juvenil, intentamos mostrar cómo es posible desarrollar los planteamientos que hemos presentado, utilizando herramientas informáticas de acceso relativamente fácil (NVivo y SPSS). Si se parte de las preguntas de investigación, la integración de métodos puede desarrollarse según tres modelos de diseños (Creswell, 2014; Creswell \& Clark, 2011): 1) diseños concurrentes (básicamente, se suman análisis realizados en paralelo); 2) diseños secuenciales (primero se trabaja con un enfoque y luego con otro) o diseños multifase (con diversas secuencias entrelazadas) y, finalmente, los diseños claramente integrados; 3) los anidados o transformadores (integrando informaciones y datos diversos en los mismos análisis). Este último tipo de diseño es el que hemos pretendido desarrollar a lo largo de las investigaciones recientes. El planteamiento de partida implica el uso de datos cuantitativos y cualitativos integrados, para obtener nuevas perspectivas y respuestas más complejas, en relación a las preguntas de investigación. Podemos, por ejemplo, preguntar cómo se ha desarrollado la parentalidad sustitutiva a lo largo de los procesos de emancipación de los jóvenes extutelados, planteando análisis de frecuencias de visitas o contactos, junto a cuestiones relativas al tipo de vínculo entre los educadores y los jóvenes. Un análisis de grupos de discusión, por ejemplo, se puede desarrollar mediante el análisis semántico (de contenido) de las intervenciones; pero, a la vez, analizar el propio grupo desde una perspectiva sociométrica (análisis micro de redes).

El conjunto de cuestiones clave para desarrollar un enfoque de análisis integrado se puede mostrar en diversos contextos de análisis. A lo largo de las dos últimas décadas hemos aplicado programas familiares y realizado análisis sobre la adolescencia y la juventud. El objetivo general de esta publicación es el análisis de los efectos del programa de competencia familiar (PCF 12-16) sobre los adolescentes y sus familias, presentado como un ejemplo de aplicación de integración de métodos.

\section{Procesos de Emancipación Juvenil de Ex-Tutelados}

Por diversas y adversas circunstancias psicológicas y/o socioculturales, son bastantes los núcleos familiares que ofrecen un modelo de referencia poco afortunado, proporcionando a los hijos diversos obstáculos para lograr el adecuado desarrollo personal y social (Bijleveld, Dedding, \& Bunders-Aelen, 2015, Gradaílle, Montserrat, \& Ballester, 2018). El acogimiento residencial ha servido como alternativa a aquellos niños y niñas que han sido declarados en desamparo y, aunque se intentan potenciar otras alternativas, como es el caso del acogimiento familiar, lo cierto es que el número de niños que permanece viviendo 
en acogimiento residencial es considerable. El acogimiento residencial puede cumplir varias funciones: la preparación para la reunificación familiar; la preparación para un acogimiento familiar permanente; trabajar la emancipación; o como lugar de estancia transitoria (Ballester, Caride, Melendro, \& Montserrat, 2016; Bijleveld et al., 2015, LlosadaGistau, Casas, \& Montserrat, 2016).

A pesar del consenso sobre la necesidad del trabajo con familias, en prevención universal y en situaciones de protección, aún se deben desarrollar mejores modelos de trabajo (Vis, Holtan, \& Thomas, 2012). La reforma legal en infancia (Leyes $8 / 2015$ y 26/2015) insiste en el sentido apuntado: la protección a la infancia se realizará mediante la prevención de situaciones de riesgo, se dará prioridad a medidas consensuadas y estables frente a las temporales, las familiares ante las institucionales.

En situaciones de tutela por la administración, es necesario disponer de un análisis de los procesos y del sistema familiar. Para desarrollar la actuación de los profesionales y para aprovechar la red de apoyo, a fin de facilitar procesos de retorno familiar o la emancipación (López \& Valle, 2016). En la preparación de estos procesos, disponer de recursos es un aspecto crítico para profesionales y servicios, pero especialmente para los adolescentes (Valle, Lázaro-Visa, López, \& Bravo, 2011).

Los programas de mejora de la competencia familiar resultan clave. El Programa de Competencia Familiar 12-16 (PCF), validado para población española, se basa en el Modelo Ecológico Social relativo al comportamiento de adolescentes y jóvenes, el cual estableció que las relaciones y la dinámica familiar son factores de riesgo o protección poderosos en la prevención (Ballester, Valero, Orte, \& Amer, 2018; Orte et al., 2015a).

\section{Integración de Métodos e Investigación de Procesos de Emancipación}

En el capítulo, se presenta la primera investigación integrada, sobre la preparación de los procesos de emancipación de tres grupos de jóvenes en el sistema de protección a la Infancia y adolescencia de Mallorca. Entendida como una aplicación del modelo de integración de métodos (Creswell, 2014; Creswell \& Clark, 2011) aplicada en los procesos de emancipación y para el fortalecimiento de las relaciones. Hipótesis: la participación en las 14 sesiones del PCF, de dos horas de duración, reforzará el funcionamiento familiar positivo, así como la calidad de las relaciones mantenidas en el núcleo familiar o el núcleo de convivencia. El programa previene posibles problemas de adaptación y ajuste psicosocial en los jóvenes de 12 a 18 años, fomenta las competencias parentales en padres y tutores, así como las habilidades de relación en los adolescentes y jóvenes, y potencia los factores de protección (Orte, Ballester, \& March, 2013; Orte et al., 2015b; Orte, Ballester, Pozo, \& Vives, 2017; Orte, Ballester, Vives, \& Amer, 2016).

Al margen de los objetivos metodológicos, los objetivos de la investigación son:

1. Analizar la eficacia de un programa de desarrollo de competencias parentales y familiares, dirigido a mejorar la convivencia.

2. Analizar la eficacia de un programa dirigido a prevenir conductas inadaptadas y de riesgo, mediante la mejora de actitudes y potenciando los factores de protección.

Los contenidos del PCF se trabajan en 14 sesiones de 2 horas, incluyendo: 1) mejora de los vínculos; 2) mejora de la comunicación; 3) mejora de la motivación, negociación, límites; 4) implicación de los miembros de la unidad de convivencia; 5) desarrollo del autocontrol; 6) mejora de la parentalidad de los padres o tutores, así como las habilidades para la vida independiente; 7) organización de la convivencia, cohesión, resiliencia; y 8) actitudes, mitos, normas parentales sobre consumo de tabaco, alcohol y otras drogas.

El análisis del programa incluye a los jóvenes, a sus familias y al propio programa, para demostrar su eficacia y revisar todos los posibles efectos del programa. 


\section{Método}

A continuación, se presentan las características más destacadas de la metodología utilizada, de acuerdo a los criterios estándar para su consideración.

\subsection{Fases de la investigación}

El estudio de la aplicación y la evaluación del programa incluye tres momentos evaluativos: a) antes del inicio del programa (selección de la muestra de familias y posterior pre-test) 2) al terminar (post-test) y 3) después de 6 meses de la implementación (seguimiento). Todo el estudio se desarrolla mediante un diseño cuasi-experimental riguroso, integrando metodologías cualitativas como la observación, los autoinformes, las imágenes de las sesiones y los informes de progreso de los participantes. Se trabaja con 3 grupos experimentales y un grupo de control. El diseño, en cuanto a la experimentación, se basa en el control de las condiciones experimentales, en las aplicaciones del programa: se eliminan los factores perturbadores, se mantienen constantes las condiciones experimentales. Se pueden resumir las fases en el siguiente gráfico 1:

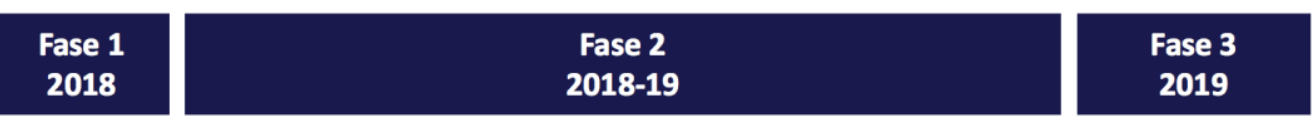

Formación de formadores (psicólogos, pedagogos, educadores o TS) Implementación del programa. Recogida y registro de datos

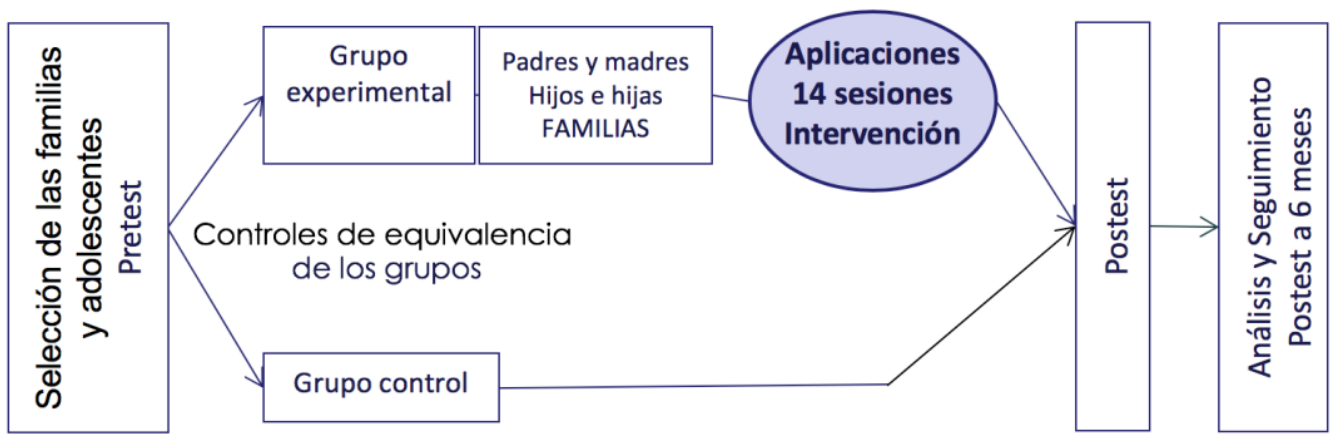

Gráfico 1: Fases del proceso de investigación

\subsection{Participantes}

La implementación del programa se realiza con 30 familias, seleccionadas a partir de cumplir las condiciones de inclusión (consentimiento informado, solicitar ayuda para mejorar relaciones, edad de los hijos tutelados por la administración, en espera de emancipación) y ninguna de las condiciones de exclusión (consumo de alcohol u otras drogas, rechazo de padres o hijos a participar). Conscientes del número de tutelados y de las circunstancias personales, familiares y contextuales de cada joven y del servicio que lo acoge, se planteó realizar 3 implementaciones en tiempo consecutivos, con 10 familias en cada caso.

La edad de los jóvenes es determinada por la normativa; por lo tanto, se ha implementado el PCF con familias con jóvenes en proceso de emancipación a partir de los 14 hasta los 18 años, pero aceptando adolescentes desde 12 años. 


\subsection{Instrumentos}

Se trabaja con cuestionarios de evaluación de la parentalidad validados para población española (Orte, Ballester, \& March, 2009,2013). También se utilizan los cuestionarios del sistema de evaluación de la conducta de niños y adolescentes (BASC) (Reynolds \& Kamphaus, 2004). Se aplica un cuestionario sobre consumo y actitudes ante las drogas y otras conductas adictivas (CAD), utilizado por el Plan Nacional Sobre Drogas (Crespo, et al., 2004; Macià, 1986; PNSD, 2015), así como un cuestionario de valoración del programa y de evaluación de diversos factores (formadores, materiales y sesiones, cambio conseguido) que incluye preguntas abiertas en un formato de entrevista cara a cara con el formador en cada centro. Estos cuestionarios están validados y pueden ser consultados (Orte, Ballester, Vives, \& Amer, 2016). Además, se incluyen cuestionarios para profesorado y educadores, referidos a alumnos o tutelados, como los registros de progreso de los participantes, basados en el registro de aportaciones para la tarea y aportaciones emocionales (Orte \& GIFES, 2016).

Todos los instrumentos evaluan aspectos cualitativos de las conductas, aunque unos se analizan desde perspectivas cuantitativas y otros cualitativas. Dos instrumentos de evaluación validados (BASC y CAD) tienen orientación cuantitativa. Las entrevistas, como es evidente, se analizan cualitativamente. El resto de instrumentos incluyen componentes cualitativos y cuantitativos.

\subsection{Análisis de la información y los dados}

Los análisis se estructuran en tres tipos de metodologías integradas, de acuerdo al cuadro que se presenta a continuación (Ballester, Nadal, \& Amer, 2017):

Cuadro 1. Análisis, métodos, instrumentos

\begin{tabular}{|c|c|c|c|}
\hline Análisis & Metodología & Instrumentos & $\begin{array}{l}\text { Programa de } \\
\text { análisis }\end{array}$ \\
\hline \multirow[t]{2}{*}{ PARTICIPACIÓN } & Observación & Registros de progreso & SPSS y NVivo \\
\hline & Observación & $\begin{array}{l}\text { Evaluación de } \\
\text { sesiones }\end{array}$ & SPSS y NVivo \\
\hline \multirow[t]{4}{*}{ IMPLEMENTACIÓN } & Observación & $\begin{array}{l}\text { Evaluación de la } \\
\text { fidelidad }\end{array}$ & SPSS y NVivo \\
\hline & Autoinforme & $\begin{array}{l}\text { Autoevaluación } \\
\text { formadores }\end{array}$ & SPSS y NVivo \\
\hline & Observación & Fotos de las sesiones & NVivo \\
\hline & Etnografía & $\begin{array}{l}\text { Registro escrito-Diario } \\
\text { de campo }\end{array}$ & NVivo \\
\hline \multirow[t]{4}{*}{ RESULTADOS } & $\begin{array}{l}\text { Cuasi- } \\
\text { Experimental }\end{array}$ & Registros pre-post & SPSS \\
\hline & $\begin{array}{l}\text { Cuasi- } \\
\text { Experimental }\end{array}$ & $\begin{array}{l}\text { Registros pre-post } \\
\text { BASC }\end{array}$ & SPSS \\
\hline & $\begin{array}{l}\text { Cuasi- } \\
\text { Experimental }\end{array}$ & $\begin{array}{l}\text { Registros pre-post } \\
\text { drogas }\end{array}$ & SPSS \\
\hline & $\begin{array}{l}\text { Cuasi- } \\
\text { Experimental }\end{array}$ & $\begin{array}{l}\text { Registros post } \\
\text { satisfacción }\end{array}$ & SPSS y NVivo \\
\hline
\end{tabular}


Se realizan análisis para contrastar hipótesis, basados en análisis de varianza, comparaciones de medias y análisis de cluster (Ballester et al., 2018; Orte, \& Ballester, 2018). Los análisis cualitativos incluyen análisis de contenido (semántico de categorías), así como análisis de redes familiares y redes de apoyo. Por motivos de espacio, solo se incluyen dos de los análisis. En NVivo se trabaja con las clasificaciones (edad, sexo, tipo de familia, etc.) recogidas en SPSS, importándolas desde NVivo. Así, los estudios de progreso de los participantes y del proceso de las aplicaciones, se enriquecen con los datos concretos de las clasificaciones.

\section{Resultados}

El análisis de la captación, retención y adherencia al programa ofrece resultados relevantes, la retención es del $96 \%$ y la adherencia del $70 \%$. En los tres casos se han desarrollado análisis cualitativos específicos sobre la respuesta a la captación realizada con el apoyo de entrevista motivacional, la retención (técnicas de implicación) y la adherencia (mejores niveles de seguimiento). Los análisis se basan en los registros de progreso de los participantes, evaluaciones de participación, cumplimentadas en cada sesión. Se sigue el modelo de los estudios follow-up (Kawatsu, Uchimura, Ohkado, \& Kato, 2018; Negreiros, Ballester, Valero, Carmo, \& Gama, 2019).

\subsection{Resultados del análisis cuantitativo}

A continuación, se presentan los resultados obtenidos a partir de uno de los análisis (ver anexo 1), empezando por cinco factores familiares informados por los padres.

El conflicto familiar. Se refiere a la baja conflictividad y las buenas relaciones. Los resultados muestran una reducción moderada y significativa ( $t=2,043 ; p=0,037)$.

Parentalidad positiva, evaluada a partir de la disciplina positiva, así como la capacidad de aportar orientación eficaz, poner límites, motivar a sus hijos y otros aspectos de la parentalidad. Los resultados muestran una mejora significativa $(t=-2,418 ; p=0,005)$.

Supervisión familiar. Incluye una amplia variedad de informaciones significativas, referidas al control en las visitas a la familia. Los resultados muestran un incremento no son suficientemente significativo $(t=-0,327 ; p=0,702)$.

Resiliencia familiar, entendida como la capacidad de la familia para hacer frente a los retos que se le plantean (salud, relaciones afectivas, apoyo en la familia, etc.) de manera efectiva. Los resultados muestran un aumento significativo $(t=-4,751 ; p=0,000)$.

Implicación familiar. Se incluyen actuaciones orientadas a mejorarla, como el desarrollo del tiempo positivo. Se observa una mejora significativa $(t=-2,554 ; p=0,006)$.

Ambiente familiar, informa sobre la capacidad de mejorar el clima de relaciones entre padres e hijos, así como entre las figuras parentales (Orte et al., 2015a). La diferencia no es significativa, a pesar de los cambios en la dirección adecuada $(t=-1,402 ; p=0,200)$.

Familia e hijos. Se consideran los cinco factores resultantes de la factorización del cuestionario para hijos. El primer factor informa de la implicación familiar, la preocupación por parte de los padres del desarrollo de sus hijos. Los resultados muestran moderado incremento no estadísticamente significativo $(p>0,05)$.

Cohesión familiar. En el cuestionario de hijos se refiere a la baja conflictividad y las buenas relaciones. Los resultados muestran una moderada mejora, $(p>0,05)$.

Control de los problemas escolares, no solo académicos sino también los relativos a conflictos. Se observa una mejora moderada, pero no significativa $(p>0,05)$.

Habilidades sociales para los hijos (hablar, escuchar activamente, autocontrol, asertividad, etc.). Se observa una mejora, no significativa estadísticamente $(p>0,05)$. 
Capacidad para poner límites y evitar problemas, también observa una mejora clara, con significación estadística ( $t=-2,332 ; p=0,009)$.

Se han producido las mejoras esperadas, aunque no con las dimensiones necesarias. A continuación, se presentan los resultados de las escalas BASC.

Escala 1. Agresividad: tendencia a actuar de forma hostil. Se ha producido una reducción de los valores, siendo estadísticamente significativo el cambio $(t=1,876 ; p=0,043)$.

Escala 2. Hiperactividad: hiperactividad e impulsividad. Se ha producido una reducción de los valores, sin llegar a ser estadísticamente significativo el cambio $(p=0,068)$.

Escala 3. Problemas de atención: evalúa la dificultad para mantener la atención y la tendencia a distraerse con facilidad ssepi:Los cambios son significativos $(t=2,532 ; p=0,017)$.

Escala 4. Depresión: valora el estado de ánimo disfórico, las ideas de suicidio, el aislamiento y los autorreproches. Los cambios son significativos ( $t=2,082 ; p=0,035)$.

Escala 5. Problemas de ansiedad: evalúa la preocupación excesiva, los miedos y fobias, la auto desaprobación. Los cambios son significativos ( $t=2,771 ; p=0,007)$.

Escala 6. Somatización: evalúa la tendencia a las quejas somáticas, no justificadas. išpe ha producido una reducción estadísticamente significativa ( $t=2,801 ; p=0,017)$.

Escala 7. Interiorizar Problemas: incluye Ansiedad, Depresión y Somatización. Los cambios, en el conjunto del factor son significativos: reducción de los valores de interiorización de los problemas ( $t=2,779 ; p=0,009)$.

De los factores informados por los hijos se han seleccionado 9, los primeros del BASC y los tres últimos del Cuestionario de Conocimiento y Actitudes ante las drogas.

Estrés social que sufren los adolescentes en las relaciones interpersonales. Se han producido reducciones, con moderada significación estadística ( $t=2,330 ; p=0,044)$.

Depresión: evalúa sentimientos como la tristeza, la soledad y el escaso disfrute ante la vida. Se observa una reducción con significación estadística ( $t=2,558 ; p=0,012)$.

Sentido de incapacidad: falta de confianza del adolescente sobre su propia capacidad. Se han reducido los valores, con resultados significativos ( $t=3,070 ; p=0,002)$.

Relaciones interpersonales: evalúa el éxito y satisfacción del adolescente en sus relaciones. Se ha producido una mejora no significativa $(t=-0,442 ; p=0,530)$.

Relaciones con los padres: se ha observado una mejora de las puntuaciones, estadísticamente significativa $(t=-2,501 ; p=0,017)$.

Autoestima. Se ha producido una mejora significativa ( $t=-2,068 ; p=0,047)$.

Disponibilidad para el consumo. Se reduce la posible aceptación de ofertas de consumo, pero no de manera significativa $(t=0,492 ; p=0,607)$. Creencias erróneas (asociadas a riesgo), se produce una reducción, no estadísticamente significativa ( $\mathrm{t}=0,285$; $\mathrm{p}=0,770$ ).

La tercera escala de actitudes: las creencias correctas o protectoras, se observa que los cambios positivos no son estadísticamente significativos $(t=-1,121 ; p=0,402)$.

\subsection{Resultados del análisis cualitativo: el análisis DAFO del PCF 12- 16}

El análisis DAFO, realizado con NVivo a partir de las entrevistas e informes de los formadores, muestra el potencial del análisis cualitativo. Las categorías se constituyen en el análisis de contenido y se asocian a las intervenciones de los profesionales. Se desarrolla un análisis de contenido semántico, identificando unidades semánticas diferenciales, de acuerdo a los centros de interés previstos en el árbol de categorías estructurado previamente, pero incorporando nuevas unidades semánticas, en función de 
categorías emergentes, de acuerdo a los modelos de análisis establecidos, para el análisis semántico, en Ballester, Orte y Oliver (2003), Ballester (2006) y Ballester (2014).

\subsubsection{Debilidades}

Se ha estructurado según cuatro categorías y 11 subcategorías.

A. Funcionamiento de la organización. 1. Rutina: Automatismos. Rutina que lleve a realizar actuaciones sin planificación o análisis. 2. Red: Debilidad de autoridad/prestigio ante otros recursos. Escasez de recursos en la Comunidad para proveer de apoyos estables, duraderos y normalizados. 3. Tiempos. Dificultades para compatibilizar el ritmo familiar con las exigencias de un programa de bastantes sesiones (14). Temporalización rígida.

B. Sistematización de la actuación. 4. Objetivos. En algunas familias, es difícil definir objetivos asumibles por los padres e hijos adolescentes. Dificultad para fijar objetivos realistas y alcanzables, teniendo en cuenta las capacidades familiares y la realidad sociocomunitaria. 5. Pronóstico. Dificultad para hacer pronósticos: identificar los indicadores clave para tomar una decisión de reunificación familiar, de recomendar sesiones individuales de apoyo.

C. Metodología del trabajo con familias. 6. Habilidades parentales: manejar ciertas características de los padres como la baja capacidad intelectual, condicionantes de las intervenciones. 7. Dificultades. Dificultad ante familias esquivas. Dificultad en intervención con personas con trastornos (inestabilidad emocional, adicciones, violencia de género). 8. Familias "difíciles". Escasas herramientas para obtener resultados óptimos en perfiles complejos. Dificultad para implicar a padres "periféricos".

D. Cambios efectivos. 9. Nuevas necesidades. Limitada capacidad para dar respuesta a necesidades emergentes. 10. Mantenimiento de los cambios. Conseguir elevar el número de familias capaces de estabilizar los cambios conseguidos. 11. Evaluación. Escaso aprovechamiento de la información basada en instrumentos validados.

\subsubsection{Amenazas}

El análisis se ha estructurado según tres categorías y 9 subcategorías.

A. Contexto institucional. 1. Pérdida de perfil. Presiones para incluir en el PCF a familias que no se ajustan al perfil. 2. Política de servicios sociales rígida. Las políticas de bienestar social colisionan con los tiempos de estas familias, las necesidades no pueden esperar meses. 3. Dificultades de red. Falta de sincronía con otras instituciones de la red que pueden dificultar el plan de actuación, en especial los centros educativos.

B. Dinámica de los equipos. 4. Implicación. Necesidad de fuerte implicación para introducir mejoras y nuevas herramientas. 5. Enfoque. Riesgo de paternalismos. Dificultad para priorizar objetivos en la intervención psicosocial con familias que "fracasan" una y otra vez. 6. Dinámica equipos. Necesidad de unificar criterios socioeducativos con familias.

C. Relación con las familias. 7. Demanda u obligación. Participantes "obligados". Sobreintervención de recursos con la misma familia. 8. Pobreza. Familias sin ingresos estables. Contexto con dificultades de establecer una red de apoyo. 9. Cronificación. Los cambios en las habilidades son lentos. Familias cronificadas en servicios sociales.

\subsubsection{Fuerzas}

El análisis se ha estructurado según dos categorías y 6 subcategorías.

A. Dinámica de los equipos. 1. Recursos del PCF 12-16. Instrumentos validados, buenas evaluaciones. 2. Experiencia y red. Experiencia de los equipos de apoyo (UIB). 
Contactos con la red mantenidos a lo largo de años. Buenas dinámicas de trabajo. 3. Implicación y trabajo en equipo. Adecuada implicación de cada profesional con los participantes. Apropiado sistema de trabajo en equipo con supervisión de psicólogas. Especialización profesional elevada en materia de intervención familiar del equipo técnico.

B. Buenas intervenciones con las familias y adolescentes. 4. Metodología. Efectividad del encuadre que se proporciona a la familia: cómo llegaron a la situación actual. Flexibilidad y adaptación de la intervención. 5. Conexión. Facilidad para conectar con adolescentes, gracias al ambiente del programa y a las dramatizaciones. Capacidad de escucha y empatía. 6. Empowerment. Capacidad para poner de relieve las capacidades y cualidades. Creencia en el potencial de cambio de las familias y adolescentes.

\subsubsection{Oportunidades}

El análisis ofrece dos categorías y 6 subcategorías.

A. La propia organización como oportunidad. 1. Capacidad del equipo. La formación y experiencia del personal profesional en intervención familiar. La capacidad para el trabajo en equipo. Preparación. 2. Dinámica interna. La supervisión efectiva y ágil. Adecuado seguimiento. 3. Programa e innovación. Ampliación del Programa según nuevas necesidades (entrevista motivacional, prevención indicada...). Investigación y formación actualizada acorde a las necesidades del Programa.

B. El entorno y la red. 4. Trabajo en red. Intervención coordinada con otros agentes sociales y servicios. 5. Entorno familiar. Apoyo de la familia extensa y/o personas de confianza. 6. Entorno social. Transferencias al entorno: la experiencia de los formadores puede externalizarse y compartirse a través de cursos y materiales para profesionales.

Como se puede ver, el análisis DAFO, realizado con NVivo, permite complementar el análisis cuantitativo y aportar una nueva visión de procesos y resultados.

\section{Discusión y conclusiones}

Una de las limitaciones observada tiene que ver con el tamaño y la selección de la muestra. Se trabaja con 30 familias, en espera de retorno familiar o emancipación de sus hijos. Cumplir todas las condiciones necesarias para poder participar en un programa de estas características, así como autorizar la participación, no es fácil de conseguir. Otra cuestión es la limitación de las recogidas de información cuantitativas y cualitativas: una parte de las dinámicas se nos escapan, ya que no se puede estar presente en las visitas familiares ni en otras situaciones de relación entre las familias y sus hijos, solo los autoinformes nos permiten acceder a algunos contenidos clave de sus procesos.

En relación al objetivo específico 1, de evaluación de la eficacia de un programa de desarrollo de competencias parentales y familiares, se comprueba que las familias que participan y finalizan el PCF, experimentan cambios positivos en sus dinámicas. Familias de alto riesgo mejoran sus dinámicas familiares, igual que las familias de menor riesgo, como se ha podido acreditar en otros estudios (Crawford y Novak, 2008; Hidalgo et al., 2012). La vulnerabilidad de la familia en contextos de protección a la infancia no determina la posibilidad del cambio de las dinámicas familiares (Ballester et al., 2018). Los factores familiares, referidos a los padres o al conjunto de la familia, más positivos son: reducción del conflicto familiar, mejora de la parentalidad positiva, mejora de la resiliencia, mejora de la implicación familiar.

Hay quien afirma que tampoco existe relación entre las dinámicas familiares previas y la eficacia del programa (Rosenmam, Goates, \& Hill, 2012; Guyll et al., 2004), si bien, sí pueden afectar a la participación y la implicación. Desde esta perspectiva, se entiende que al margen de cuál sea el nivel previo de las familias, los programas de prevención familiar pueden producir efectos positivos (Ballester et al.,2018; Hidalgo et al., 2012). 
Por lo que respecta al objetivo específico 2 , relativo a la eficacia del programa dirigido a prevenir conductas inadaptadas y de riesgo, mediante la mejora de sus actitudes y potenciando los factores de protección; el PCF para adolescentes (12-18) demuestra obtener buenos resultados, con adolescentes en situaciones de difícil convivencia (aún no se ha recuperado la relación de convivencia normalizada), con un conjunto apreciable de los resultados. Los cambios referidos a los adolescentes, más positivos, son: reducción del estrés, los síntomas asociados a la depresión y el sentido de incapacidad; así como la mejora de la relación con los padres y la mejora de la autoestima. Por lo que se refiere a la prevención centrada en la mejora de las actitudes ante el consumo de sustancias, los cambios no son significativos, aunque se observen mejoras.

A partir de los resultados identificados, se pueden considerar una serie de cuestiones:

1. El PCF, aplicado con adolescentes en preparación de los procesos de retorno familiar o emancipación, ha mostrado su eficacia para mantener el compromiso de los participantes, consiguiendo un nivel de retención de la muestra importante.

2. La integración de métodos es aplicable en la evaluación de la eficacia, incluyendo la evaluación de procesos (enfoque cualitativo) y resultados (enfoque cuantitativo).

3. En todos los programas socioeducativos con familias quedan diversas cuestiones importantes por aclarar sobre sus efectos a más largo plazo; en lo que se refiere al PCF: cuáles son los efectos a largo plazo de los programas y cuáles son los factores que más influyen en el mantenimiento o pérdida de efectos; cómo interacciona el PCF con otros cambios evolutivos y con los que se producen en las familias cuando se produce el retorno familiar o la emancipación con apoyo de la familia.

\subsection{Orientaciones para futuros estudios y aplicabilidad en otros contextos}

El trabajo presentado, aporta diversos aprendizajes:

1) Sobre las metodologías integradas. Realizar investigación evaluativa, basada en modelos cuasi-experimentales, sin complementarlas con modelos cualitativos, empobrece los análisis. La conexión concreta que hemos probado, mediante las clasificaciones (SPSS) importadas en NVivo, conectan los dos tipos de información. La integración de métodos, mediante la conexión del análisis de procesos (cualitativos) y de resultados (cuantitativos), enriquece el diseño cuasi-experimental.

2) Modelos de investigación participativa. Las evaluaciones para obtener evidencias científicas, tienen el peligro de los sesgos. Deben limitarse los procesos participativos relativos a la metodología. Sin embargo, dicha pérdida de participación se puede compensar incrementándola en el proceso de implementación del programa: preparación de materiales didácticos, actividades sociales del programa, etc.

3) La manualización completa del programa permite un incremento de la transparencia, respondiendo por adelantado a las dudas sobre en qué consiste el programa, así como sobre la posible pérdida de eficacia por la aplicación poco rigurosa del mismo.

En cuanto a la aplicabilidad, se deben destacar dos cuestiones complementarias.

1) Impacto sobre las políticas públicas. Se trabaja en el reconocimiento de la línea de intervención que representa este programa familiar. Se puede mostrar un efecto en la mejora de las dinámicas de las familias de los jóvenes, pero también en su calidad de vida y su bienestar subjetivo, junto con el de su familia, restableciendo los vínculos posibles y (re)convirtiéndola en una fuente protectora.

2) La investigación sobre la eficacia de los programas familiares, en la preparación de la emancipación, utilizando integración de métodos, se puede replicar, ya que la metodología, técnicas, instrumentos y análisis concretos están a la disposición de quien quiera conocerlos. 


\section{Referencias}

Ballester, L., Orte, C., \& Oliver, J.L. (2003). Análisis cualitativo de entrevistas. Nómadas, (18), 140149.

Ballester, L. (2006). El análisis semántico y pragmático de las entrevistas de investigación. EMPIRIA. Revista de Metodología de las ciencias sociales, (11), 107-129.

Ballester, L. (2014). Principios del análisis semántico. En: Sarabia, F. J. Métodos de investigación social y de la empresa. Ediciones Pirámide. Pp. 673-691.

Ballester, L., Caride, J.A., Melendro, M., \& Montserrat, C. (2016). Jóvenes que construyen futuros: de la exclusión a la inclusión social. Santiago de Compostela: Ediciones de la Universidad de Santiago.

Ballester, L., Nadal, A., \& Amer, J. (2017). Métodos y técnicas de investigación educativa. Palma: Ediciones UIB.

Ballester, L., Valero, M., Orte, C., \& Amer, J. (2018). An analysis of family dynamics: a selective substance abuse prevention programme for adolescents. European Journal of Social Work, 23(1), 93-105. https://doi.org/10.1080/13691457.2018.1473842

Bijleveld, G., Dedding, C., \& Bunders-Aelen, J. (2015). Children's and Young people's participation within child welfare and child protection services: A state-of-the art review. Child \& Family Social Work, 20(2), 129-138. https://doi.org/10.1111/cfs.1282

Brannen, J. (2017). Mixing methods: Qualitative and quantitative research. Routledge.

Crawford, L.A., \& Novak, K.B. (2008). Parent-child relations and peer associations as mediators of the family structure substance use relationship. Journal of Family Issues, 29(2), 155-184. https://doi.org/10.1177/0192513X07304461

Crespo, J.L.C., Rodríguez, O.G., Villa, R.S., Hermida, J.R.F., Cueto, E.G., Pérez, J.M.E., \& Díaz, S.A.H. (2004). Construcción y validación de un cuestionario de factores de riesgo interpersonales para el consumo de drogas en la adolescencia. Psicothema, 16(4), 674-679.

Creswell, J. W. (2014). Research design. 4th edition. London: Sage publications.

Creswell, J.W., \& Clark, V.L.P. (2011). Designing and conducting mixed methods research. Thousand Oaks, California: Sage publications.

Creswell, J.W., \& Creswell, J.D. (2017). Research design: Qualitative, quantitative, and mixed methods approaches. London: Sage publications.

Denzin, N.K., \& Lincoln, Y.S. (Eds.). (2011). The SAGE handbook of qualitative research. London: Sage publications.

Gibbs, G. (2012). El análisis de datos en investigación cualitativa. Madrid: Morata.

Gradaílle, R., Montserrat, C., \& Ballester, L. (2018). Transition to adulthood from foster care in Spain: A biographical approach. Children and Youth Services Review, 89, 54-61. https://doi.org/10.1016/j.childyouth.2018.04.020

Guyll, M., Spoth, R.L., Chao, W., Wickrama, K.A.S., y Russell, D. (2004). Family focused preventive interventions: Evaluating parental risk moderation of substance use trajectories. Journal of Family Psychology, 18(2), 293-301. 10.1037/0893-3200.18.2.293

Halcomb, E., \& Hickman, L. (2015). Mixed methods research. 29(32), 41-47. $\underline{10.7748 / \mathrm{ns} .29 .32 .41 . e 8858}$

Hidalgo, M.V., Lorence, B., Pérez, J., \& Menéndez, S. (2012). Typology of families at psychosocial risk: The role of family structure. Revista Mexicana de Psicología, 29(2), 165-174.

Jason, L., \& Glenwick, D. (Eds.). (2016). Handbook of methodological approaches to communitybased research: Qualitative, quantitative, and mixed methods. Oxford university press.

Kawatsu, L., Uchimura, K., Ohkado, A., \& Kato, S. (2018). A combination of quantitative and qualitative methods in investigating risk factors for lost to follow-up. PloS one, 13(6). 10.1371/journal.pone.0198075 
León, O.G., \& Montero, I. (2015). Métodos de investigación en Psicología y Educación. Las tradiciones cuantitativa y cualitativa. Madrid, McGraw Hill.

Llosada-Gistau, J., Casas, F., \& Montserrat, C. (2016). What matters in for the subjective well being of children in care? Child Indicators Research, 10(3), 735-760. 10.1007/s12187-016-9405-Z

López, M., \& Valle, J.F. (2016). Foster carer experience in Spain: Analysis of the vulnerabilities of a permanent model. Psicothema, 28(2), 122-129. 0.7334/psicothema2015.168

Macià, D. (1996). Cuestionario de actitudes y Cuestionario de información. Método Conductual de Prevención de Drogodependencias. Valencia: Promolibro.

Miles, M.B., Hubermas, A.M., \& Saldaña, J. (2014). Qualitative data analysis. An expanded sourcebook. Thousand Oaks, CA: Sage publications.

Negreiros, J., Ballester, L., Valero, M., Carmo, R., \& Gama, J. (2019). A systematic review of participation in prevention family programs. Pedagogía social, 34, 63-75. https://doi.org/10.7179/PSRI 2019.34.05

Orte, C. \& GIFES (2016). Manual de implementación del Programa de Competencia familiar (1216). Palma: Universitat de les Illes Balears.

Orte, C., Ballester, L., \& March, M. (2009). Validación de la adaptación española de los cuestionarios para evaluar la competencia parental en el contexto del Programa de Competencia Familiar (PCF) (2008-2009). In XIV Congreso Nacional de Modelos de Intervención Educativa. Huelva: AIDIPE.

Orte, C., Ballester, L., \& March, M. (2013). The family competence approach. An experience of socio-educational work with families. Pedagogía Social, 21(2), 3-27. 10.7179/PSRI 2013.21.1

Orte, C., Ballester, L., March, M.X., Amer, J., Vives, M., \& Pozo, R. (2015a). The Strengthening Families Program in Spain: A long-term evaluation. Journal of Children's Services, 10(2), 101-119. https://doi.org/10.1108/JCS-03-2013-0010

Orte, C., Ballester, L., March, M.X., Oliver, J.L., Pascual, B., \& Gomila, M.A. (2015b). Development of prosocial behaviour in children after the improvement of family competences. Journal of Children's Services, 10(2), 161-172. 10.1108/JCS-02-2014-0013

Orte, C., Ballester, L., Pozo, R., \& Vives, M. (2017). Maintaining changes in an evidence-based family prevention program. A longitudinal study of families. Pedagogia Social, (29), 109-122. https://doi.org/10.7179/PSRI 2017.29.08

Orte, C., Ballester, L., Vives, M., \& Amer, J. (2016). Quality of implementation in an evidence-based family prevention program: "The Family Competence Program". Psychosocial Intervention, 25(2), 95-101. http://dx.doi.org/10.1016/j.psi.2016.03.005

Orte, C., \& Ballester, L. (2018). Intervenciones efectivas en prevención familiar de drogas. Madrid: Octaedro.

Plan Nacional Sobre Drogas. (2015). Consumo, percepciones y opiniones ante las drogas. Encuesta sobre Alcohol y Drogas en España (EDADES). Madrid: Ministerio de Sanidad, Servicios Sociales e Igualdad.

Rapley, T. (2014). Los análisis de la conversación, del discurso y de documentos en investigación cualitativa. Madrid: Morata.

Reynolds, C.R., \& Kamphaus, R.W. (2004). BASC, Sistema de evaluación de la conducta de niños $y$ adolescentes. Madrid: TEA Ediciones.

Rosenman, R. E., Goates, S., \& Hill, L. (2012). Participation in universal prevention programmes. Applied Economics, 44(1/3), 219-228. https://doi.org/10.1080/00036846.2010.502111

Valle, J.F., Lázaro-Visa, S., López, M., \& Bravo, A. (2011). Leaving family care. Transitions to adulthood from kinship care. Children and Youth Services Review, 33, 2475-2481. 10.1016/j.childyouth.2011.08.015

Vis, S.A., Holtan, A., \& Thomas, N. (2012). Obstacles for child participation in care and protection cases - why Norwegian social workers find it difficult. Child Abuse Review, 21(1), 7. $\underline{10.1002 / \text { car. } 1155}$ 
Wyse, D., Selwyn, N., Smith, E., \& Suter, L.E. (Eds.). (2016). The BERA/SAGE handbook of educational research. London: Sage publications.

\section{Apéndice}

Tabla 1- Diferencias de medias en los factores evaluados por padres y adolescentess

\begin{tabular}{|c|c|c|c|c|c|c|c|c|}
\hline \multirow{3}{*}{$\begin{array}{l}\text { FACTORES } \\
\text { EVALUADOS }\end{array}$} & \multicolumn{2}{|c|}{$\begin{array}{l}\text { Prueba Levene } \\
\text { igualdad de } \\
\text { varianzas }\end{array}$} & \multicolumn{6}{|c|}{ Prueba t para la igualdad de medias } \\
\hline & \multirow{2}{*}{$\mathbf{F}$} & \multirow{2}{*}{ Sig. } & \multirow{2}{*}{$\mathbf{t}$} & \multirow{2}{*}{ gl } & \multirow{2}{*}{$\begin{array}{c}\text { Sig. } \\
\text { (bilateral) }\end{array}$} & \multirow{2}{*}{$\begin{array}{l}\text { Diferencia } \\
\text { de medias }\end{array}$} & \multicolumn{2}{|c|}{$95 \%$ de $\mathrm{Cl}$} \\
\hline & & & & & & & Inferior & Superior \\
\hline $\begin{array}{l}\text { PADRES y MADRES } \\
\text { sobre FAMILIA } \\
\text { Conflicto familiar }\end{array}$ & 0,655 & 0,42 & 2,043 & 42 & 0,037 & $-0,4683$ & $-3,3585$ & 2,4219 \\
\hline $\begin{array}{l}\text { PADRES y MADRES } \\
\text { sobre FAMILIA } \\
\text { Supervisión }\end{array}$ & 3,533 & 0,063 & $-0,327$ & 42 & 0,702 & 3,245 & $-9,7309$ & 16,2209 \\
\hline $\begin{array}{l}\text { PADRES y MADRES } \\
\text { sobre FAMILIA } \\
\text { Resistencia y } \\
\text { Comunicación } \\
\text { familiar }\end{array}$ & 4,383 & 0,039 & $-4,751$ & 42 & 0 & $-0,5583$ & $-5,4975$ & 4,3808 \\
\hline $\begin{array}{l}\text { PADRES y MADRES } \\
\text { sobre FAMILIA } \\
\text { Implicación familiar }\end{array}$ & 3,533 & 0,063 & $-2,554$ & 42 & 0,006 & 3,245 & $-9,7309$ & 16,2209 \\
\hline $\begin{array}{l}\text { PADRES y sobre } \\
\text { FAMILIA Ambiente } \\
\text { familiar }\end{array}$ & 2,07 & 0,153 & $-1,402$ & 42 & 0,2 & $-1,4583$ & $-3,7159$ & 0,7993 \\
\hline
\end{tabular}

\begin{tabular}{|c|c|c|c|c|c|c|c|c|}
\hline \multirow{3}{*}{$\begin{array}{l}\text { FACTORES } \\
\text { EVALUADOS }\end{array}$} & \multicolumn{2}{|c|}{$\begin{array}{c}\text { Prueba Levene } \\
\text { igualdad de } \\
\text { varianzas }\end{array}$} & \multicolumn{6}{|c|}{ Prueba t para la igualdad de medias } \\
\hline & \multirow{2}{*}{$\mathbf{F}$} & \multirow{2}{*}{ Sig. } & \multirow{2}{*}{$t$} & \multirow{2}{*}{ gl } & \multirow{2}{*}{$\begin{array}{c}\text { Sig. } \\
\text { (bilateral) }\end{array}$} & \multirow{2}{*}{$\begin{array}{l}\text { Diferencia } \\
\text { de medias }\end{array}$} & \multicolumn{2}{|c|}{$95 \%$ de $\mathrm{Cl}$} \\
\hline & & & & & & & Inferior & Superior \\
\hline $\begin{array}{l}\text { ADOLESCENTES } \\
\text { Implicación familiar }\end{array}$ & 1,316 & 0,254 & 0,287 & 29 & 0,775 & 0,6138 & $-3,6327$ & 4,8604 \\
\hline $\begin{array}{l}\text { ADOLESCENTES } \\
\text { Cohesión familiar }\end{array}$ & 2,159 & 0,145 & $-0,553$ & 29 & 0,581 & $-0,4298$ & $-1,9715$ & 1,1119 \\
\hline $\begin{array}{l}\text { ADOLESCENTES } \\
\text { Control de los } \\
\text { problemas en la } \\
\text { escuela }\end{array}$ & 4,259 & 0,042 & $-0,315$ & 29 & 0,753 & $-0,1667$ & $-1,2163$ & 0,883 \\
\hline $\begin{array}{l}\text { ADOLESCENTES } \\
\text { Habilidades sociales } \\
\text { Conocimientos de } \\
\text { los adolescentes }\end{array}$ & 0,069 & 0,794 & 0,322 & 29 & 0,748 & 0,7269 & $-3,7489$ & 5,2027 \\
\hline $\begin{array}{l}\text { ADOLESCENTES } \\
\text { Capacidad para } \\
\text { poner límites y evitar } \\
\text { los problemas }\end{array}$ & 4,077 & 0,046 & $-2,332$ & 29 & 0,009 & 0,0227 & $-0,975$ & 1,0204 \\
\hline
\end{tabular}




\begin{tabular}{|c|c|c|c|c|c|c|c|c|}
\hline \multirow{3}{*}{$\begin{array}{l}\text { FACTORES } \\
\text { EVALUADOS }\end{array}$} & \multicolumn{2}{|c|}{$\begin{array}{c}\text { Prueba Levene } \\
\text { igualdad de } \\
\text { varianzas }\end{array}$} & \multicolumn{6}{|c|}{ Prueba t para la igualdad de medias } \\
\hline & \multirow{2}{*}{$\mathbf{F}$} & \multirow{2}{*}{ Sig. } & \multirow{2}{*}{$t$} & \multirow{2}{*}{ gl } & \multirow{2}{*}{$\begin{array}{c}\text { Sig. } \\
\text { (bilateral) }\end{array}$} & \multirow{2}{*}{$\begin{array}{l}\text { Diferencia } \\
\text { de medias }\end{array}$} & \multicolumn{2}{|c|}{$95 \%$ de $\mathrm{Cl}$} \\
\hline & & & & & & & Inferior & Superior \\
\hline $\begin{array}{l}\text { PADRES y MADRES } \\
\text { Hiperactividad Punt } \\
\mathrm{T}\end{array}$ & 1,451 & 0,23 & 1,04 & 42 & 0,068 & 2,5035 & $-2,2578$ & 7,2648 \\
\hline $\begin{array}{l}\text { PADRES y MADRES } \\
\text { Prob atencion Punt } T\end{array}$ & 0,103 & 0,749 & 2,532 & 42 & 0,017 & 4,1145 & 0,4475 & 7,7816 \\
\hline $\begin{array}{l}\text { PADRES y MADRES } \\
\text { Depresion Punt T }\end{array}$ & 5,096 & 0,026 & 2,082 & 42 & 0,035 & 5,365 & 0,2956 & 10,4345 \\
\hline $\begin{array}{l}\text { PADRES y MADRES } \\
\text { Ansiedad Punt T }\end{array}$ & 0,895 & 0,346 & 2,771 & 42 & 0,007 & 3,5594 & 0,1823 & 6,9364 \\
\hline $\begin{array}{l}\text { PADRES y MADRES } \\
\text { Somatización Punt T }\end{array}$ & 2,141 & 0,146 & 2,801 & 42 & 0,017 & 5,8503 & 1,2942 & 10,4063 \\
\hline $\begin{array}{l}\text { PADRES y MADRES } \\
\text { Int problemas Punt } T\end{array}$ & 2,141 & 0,146 & 2,779 & 42 & 0,009 & 5,1432 & 0,5593 & 9,727 \\
\hline
\end{tabular}

\begin{tabular}{|c|c|c|c|c|c|c|c|c|}
\hline \multirow{3}{*}{$\begin{array}{l}\text { FACTORES } \\
\text { EVALUADOS }\end{array}$} & \multicolumn{2}{|c|}{$\begin{array}{l}\text { Prueba Levene } \\
\text { igualdad de } \\
\text { varianzas }\end{array}$} & \multicolumn{6}{|c|}{ Prueba t para la igualdad de medias } \\
\hline & \multirow{2}{*}{$\mathbf{F}$} & \multirow{2}{*}{ Sig. } & \multirow{2}{*}{$t$} & \multirow{2}{*}{ gl } & \multirow{2}{*}{$\begin{array}{c}\text { Sig. } \\
\text { (bilateral) }\end{array}$} & \multirow{2}{*}{$\begin{array}{l}\text { Diferencia } \\
\text { de medias }\end{array}$} & \multicolumn{2}{|c|}{$95 \%$ de $\mathrm{Cl}$} \\
\hline & & & & & & & Inferior & Superior \\
\hline $\begin{array}{l}\text { AUTOINF } \\
\text { ADOLESCENTES } \\
\text { Depresion }\end{array}$ & 0,02 & 0,886 & 2,558 & 29 & 0,012 & 1,4745 & $-4,0045$ & 6,9535 \\
\hline $\begin{array}{l}\text { AUTOINF } \\
\text { ADOLESCENTES } \\
\text { Sentido incapacidad }\end{array}$ & 3,26 & 0,073 & 3,07 & 29 & 0,002 & 0,5385 & $-3,6943$ & 4,7712 \\
\hline $\begin{array}{l}\text { AUTOINF } \\
\text { ADOLESCENTES Rel } \\
\text { interpers }\end{array}$ & 0,244 & 0,622 & $-0,442$ & 29 & 0,53 & $-0,0684$ & $-3,8532$ & 3,7164 \\
\hline $\begin{array}{l}\text { AUTOINF } \\
\text { ADOLESCENTES Rel } \\
\text { padres }\end{array}$ & 0,005 & 0,945 & $-2,501$ & 29 & 0,017 & $-1,8565$ & $-6,9757$ & 3,2627 \\
\hline $\begin{array}{l}\text { AUTOINF } \\
\text { ADOLESCENTES } \\
\text { Autoestima }\end{array}$ & 1,572 & 0,212 & $-2,068$ & 29 & 0,047 & $-0,0249$ & $-4,3158$ & 4,2659 \\
\hline
\end{tabular}

\begin{tabular}{|c|c|c|c|c|c|c|c|c|}
\hline \multirow{3}{*}{$\begin{array}{l}\text { FACTORES } \\
\text { EVALUADOS }\end{array}$} & \multicolumn{2}{|c|}{$\begin{array}{l}\text { Prueba Levene } \\
\text { igualdad de } \\
\text { varianzas }\end{array}$} & \multicolumn{6}{|c|}{ Prueba t para la igualdad de medias } \\
\hline & \multirow{2}{*}{$\mathbf{F}$} & \multirow{2}{*}{ Sig. } & \multirow{2}{*}{$t$} & \multirow{2}{*}{ gl } & \multirow{2}{*}{$\begin{array}{c}\text { Sig. } \\
\text { (bilateral) }\end{array}$} & \multirow{2}{*}{$\begin{array}{l}\text { Diferencia } \\
\text { de medias }\end{array}$} & \multicolumn{2}{|c|}{$95 \%$ de $\mathrm{Cl}$} \\
\hline & & & & & & & Inferior & Superior \\
\hline $\begin{array}{l}\text { AUTOINF } \\
\text { ADOLESCENTES } \\
\text { Disponibilidad para } \\
\text { el consumo }\end{array}$ & 0,26 & 0,611 & 0,492 & 29 & 0,607 & 0,4957 & $-4,1272$ & 5,1185 \\
\hline $\begin{array}{l}\text { AUTOINF } \\
\text { ADOLESCENTES } \\
\text { Creencias erróneas } \\
\text { AUTOINF } \\
\text { ADOLESCENTES } \\
\text { Creencias correctas }\end{array}$ & 0,146 & 0,166 & 0,285 & 29 & 0,402 & 0,7231 & $\begin{array}{l}-3,4666 \\
-4,0742\end{array}$ & 4,9127 \\
\hline
\end{tabular}




\section{Notas biográficas}

Lluís Ballester Brage. Doctor en Sociología (UAB) y en Filosofía (UIB). Profesor de Métodos de Investigación y Análisis de Datos en la Facultad de Educación de la UIB. Subdirector del Departamento de Pedagogía y Didácticas Específicas de la UIB. Miembro del Grupo de Investigación y Formación Educativa y Social (GIFES). Investigador principal, junto a la profesora Carmen Orte, del proyecto de investigación EDU2016-79235-R - "Validación del Programa de Competencia Familiar Universal 10-14, PCF-U", 2017-2019. https://www.researchgate.net/profile/Lluis_Brage

Victòria Quesada Serra. Doctora en Ciencias Sociales por la Universidad de Cádiz. Licenciada en Pedagogía (UIB) y máster en Orientación y Evaluación SocioEducativas (UCA). De 2010 a 2017 trabajó en el área de Métodos de investigación y Diagnóstico en Educación de la UCA. Desde 2017 es profesora de Métodos de Investigación en la Universitat de les Illes Balears y miembro del Grupo de investigación y Formación Educativa y Social (GIFES). Sus líneas de investigación giran en torno a la evaluación y los programas familiares.

https://www.researchgate.net/profile/Victoria_Quesada_Serra

Albert Cabellos Vidal. Investigador (FPU) en el Departamento de Pedagogía y Didácticas Específicas de la Universidad de las Islas Baleares. Miembro del Grupo de Investigación y Formación Educativa y Social (GIFES) y del LIFAC. Sus líneas de investigación se centran en los procesos de emancipación juvenil. Graduado en Educación social por la UIB, ha trabajado como educador con adolescentes en riesgo de exclusión social y escolar en el programa de escolaridad compartida ALTER Palma.

Carlos Rosón Varela. Profesor de la Escuela de Trabajo Social de la Universidad de Santiago de Compostela. Director del Centro FAIA de Documentación e Investigación Social. Director de Igaxes. Ha participado en diversas investigaciones orientadas a la familia, infancia y juventud en riesgo de exclusión: "Estrategias eficaces de intervención socioeducativa con adolescentes en riesgo de exclusión" (2014), "Jóvenes que construyen futuros: de la exclusión a la inclusión social" (2016), "Nueva pornografía y cambios en las relaciones interpersonales" (2019), "Pornografía y educación afectivosexual" (2020). Ha coordinado varios simposios y presentado diversas comunicaciones alrededor de estos temas en diferentes Congresos Internacionales. 